\title{
A RED ALERT! NOSOCOMIAL INFECTION BY SERRATIA MARCESCENS
}

Dipankar Paul ${ }^{1}$, Indrani Bhattacharyya ${ }^{2}$, Mandira Chakraborty 3 , Palash Das ${ }^{4}$, Sangita Das Ghosh ${ }^{5}$

\section{HOW TO CITE THIS ARTICLE:}

Dipankar Paul, Indrani Bhattacharyya, Mandira Chakraborty, Palash Das, Sangita Das Ghosh. "A Red Alert! Nosocomial Infection by Serratia Marcescens". Journal of Evolution of Medical and Dental Sciences 2014; Vol. 3, Issue 49, October 02; Page: 11766-11770, DOI: 10.14260/jemds/2014/3546

INTRODUCTION: Serratia marcescens is a member of the Entero bactereacae family. S. marcescens is widely distributed in nature. It is isolated as saprophytes from soil and water. Reports of nosocomial infections caused by S. marcescens have increased in recent years. Infections include UTI, RTI, Septicaemia, Meningitis and Endotoxic shock. Only small proportion $(<10 \%)$ of strains responsible for infection are pigmented. It is the most common clinical isolate among Serratia ${ }^{2}$.We isolated Serratia marcescens from blood culture of a hospitalized patient who was hospitalized for diabetes mellitus and chronic diarrhea.

PATIENT PROFILE: A 32 yr old male, weighing 29 kg only, residing at Kolkata, admitted for last 26 days with complaints of chronic diarrhea for last $1 \mathrm{yr}$, fever for last 12 days, difficulty in opening the mouth and malnutrition. Patient is a known diabetic for last $1 \mathrm{yr}$. Clinical diagnosis is Diabetes Mellitus, chronic diarrhea and sub mucosal fibrosis. Patient was hypotensive (BP-90/60mmHg) on admission.

Upper G.I. endoscopy and colonoscopy shows no macroscopic abnormality except fundal gastritis. USG abdomen shows normal study. LFT-normal study, FBS-213mg/dl, Hb-11.8gm, TLC4500/cumm;N33 L57, Na+135mmol/L;K+ 3.6mmol/L, CD4 count- 533, HIV I \& HIV2 - Non-reactive.

MATERIALS AND METHODS: Blood culture was sent in Trypticase Soy broth. It was incubated aerobically at 370c. On 5th day turbidity developed and inoculum sub cultured on Mac Conkey and blood agar and incubated aerobically at 370c for 24 hours. On Mac Conkey agar convex, effuse, colorless colonies with irregular cremated edges developed, which became pink after 48 hours- a late lactose fermenter. On blood agar convex, raised, grayish colonies with narrow zone of haemolysis was seen.

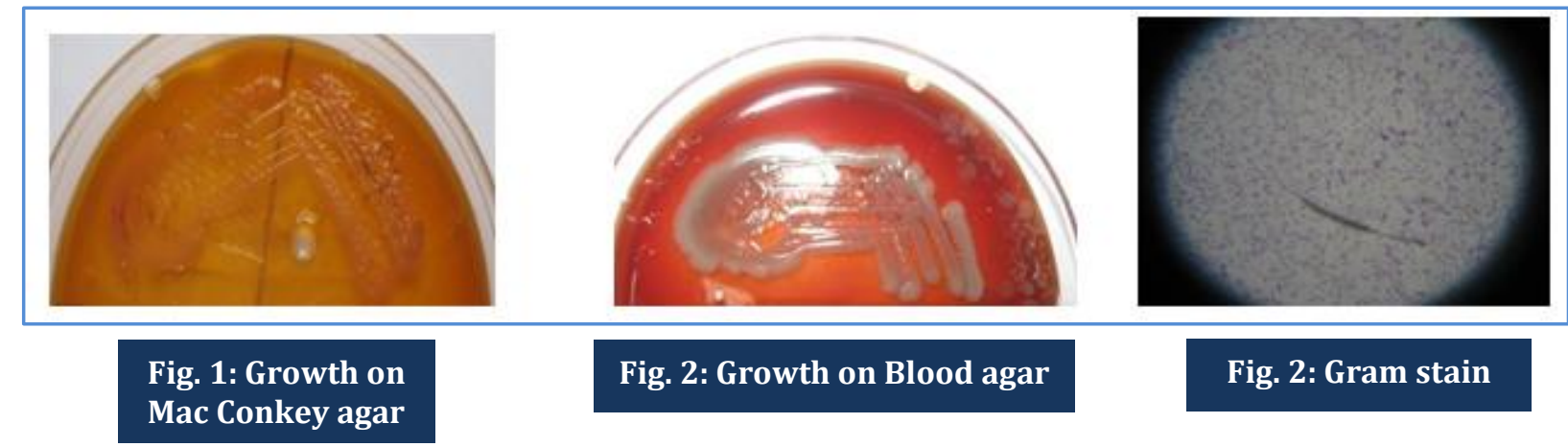

Gram stain shows small gram negative cocco bacilli. Hanging drop preparation shows motile organism. 


\section{CASE REPORT}

BIOCHEMICAL TESTS: TSI-K/A, no H2S, Indole- Neg, Urease -Neg, Citrate +ve, Oxidase -Neg, Lysine +ve, Ornithine +ve, Arginine - Neg, PPA - Neg, Reduced nitrate to nitrite, ONPG +ve, Glucose- Acid, no gas, Sucrose-Acid, no gas, Mannitol-Acid, no gas.
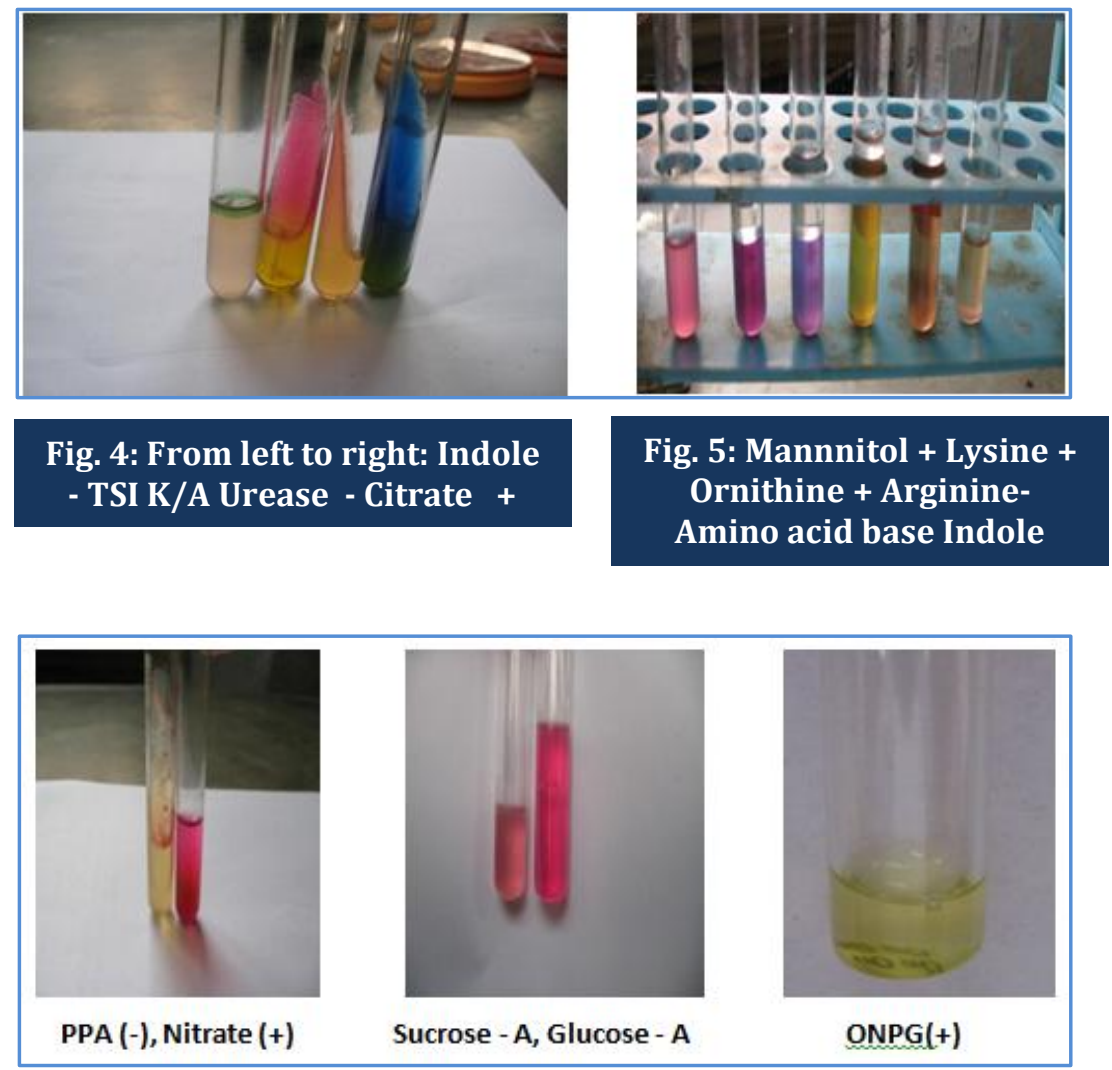

Fig. 6

Morphology, colony characters and biochemical tests are indicative of Serratia marcescens. ${ }^{3,6}$ We inoculated two nutrient agar tubes and kept one of them in room temperature and another one at $370 \mathrm{C}$ for pigment production. Abundant red non- diffusible pigment developed on nutrient agar kept in air at room temperature. There was less pigment production at 370c. The pigment was soluble in absolute alcohol, acetone, chloroform but insoluble in water. ${ }^{4}$

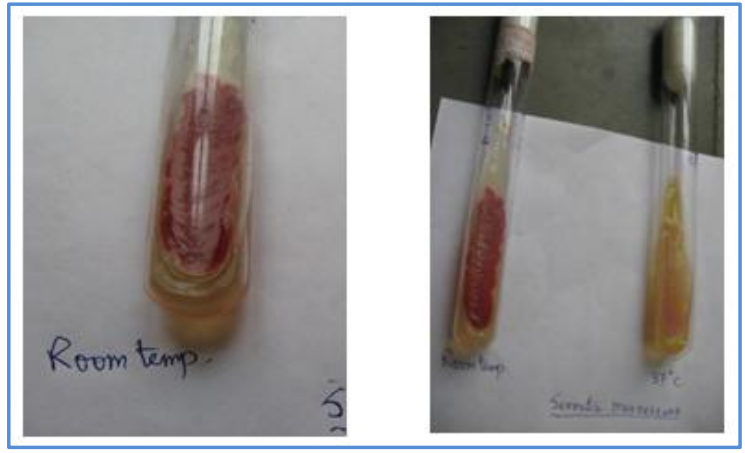

\section{Fig. 7: Pigment production at room temperature $\&$ at $37^{\circ} \mathrm{C}$}




\section{CASE REPORT}

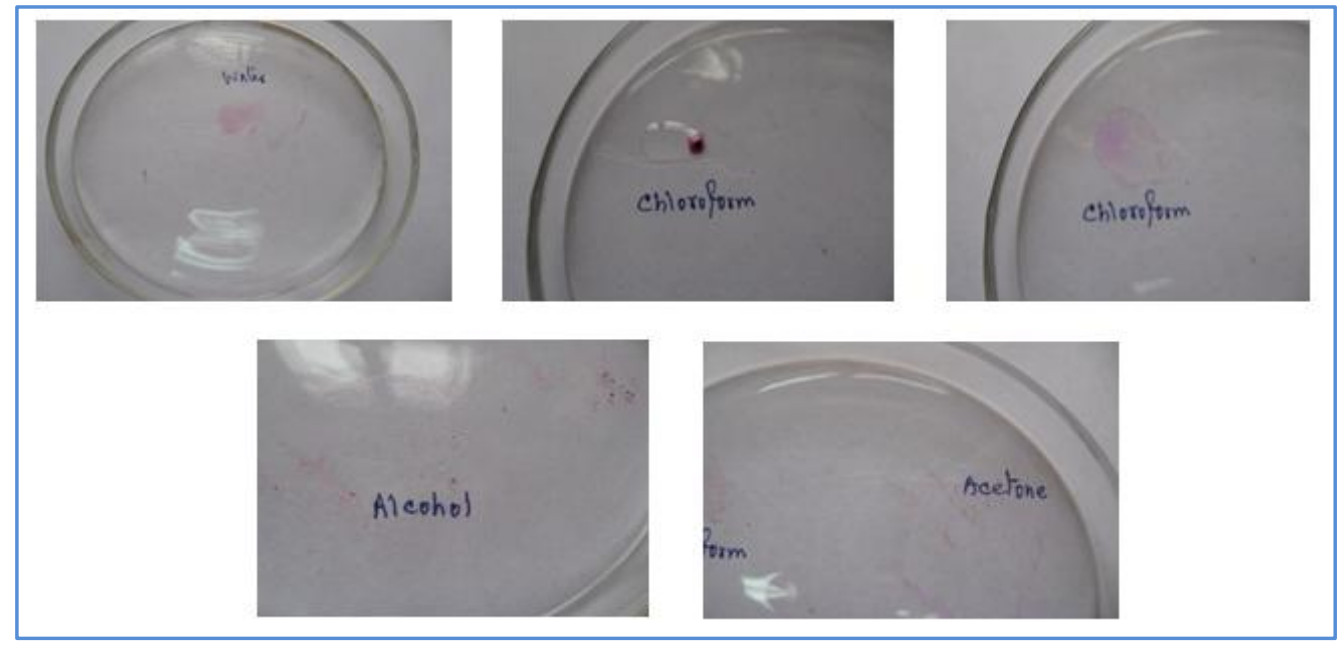

\section{Fig. 8: Pigment solubility tests}

Based on Gram-stain, morphology, motility, colony character, biochemical tests, pigment production and character of the pigment the organism is diagnosed as Serratia marcescens.

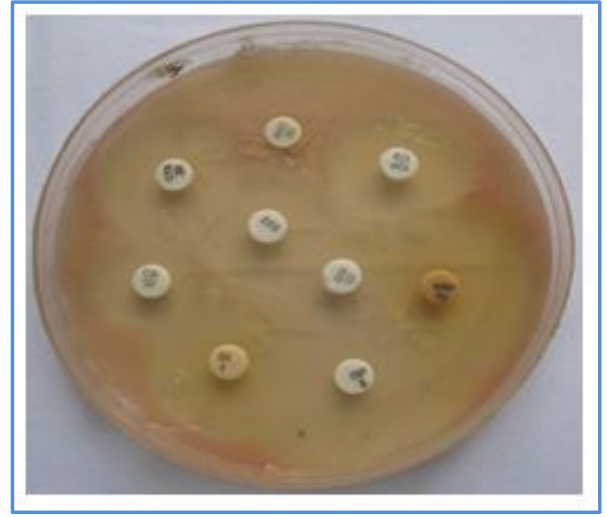

Fig. 9

Fig. 9: Drug susceptibility test on Mueller-Hinton agar with Disk diffusion method also shows nondiffusible red pigmentation and the following sensitivity pattern:

SENSITIVE TO: Pipericillin+Tazobactum Imipenem, Meropenem, Gentamycin, Ciprofloxacin, CoTrimoxazole.

RESISTANT TO: Co-amoxyclav, Ceftazidime.

TREATMENT HISTORY: Patient was on I.V. fluid, Inj. Insulin subcutaneously, Inj. Ceftriaxone \& Metronidazole intravenously. Later inj. Meropenem was added. Patient was responding to treatment. 
DISCUSSION: S. marcescens was first described and named by Bizio in 1823. Gaughran (1969) assumed that the first recorded account of Serratia marcescens dates back to 332 B.C. 1. This organism typically grows on grains such as polenta (corn), producing its characteristic red pigment. So the superstition of a supernatural force causing "bright red blood" to appear on grains spontaneously can be traced in recorded history, way before bacteriological era. The production of the red pigment prodigiosin by S. marcescens as the explanation of 'bleeding polenta' came over 2000 years later.

Genus Serratia contains 9 species. Most strains of S. plymuthica, S rubidaea (syn. S marinorubra) and some strains of $S$ marcescens produce magenta red pigment (prodigiosin) but former two species are negative for lysine and ornithine decarboxylase S. odorifera is a nonpigmented species which is lysine positive but has a characteristic musty potato like odour which aids its recognition in the laboratory. ${ }^{2}$ Some strains of S. marcescens may grow in chlorhexidine based disinfecting solution. Most of the strains are resistant to cephalosporins. Such resistance is frequently plasmid mediated and readily transferrable to other members of the Enterobacteriaceae. ${ }^{5}$

CONCLUSION: Our index patient was admitted in our institute with Diabetes mellitus, chronic diarrhoea and malnutrition and developed fever after 14 days of stay in hospital. Blood culture on 16th day of hospital stay showed bacteremia with Serratia marcescens. This is suggestive of nosocomial infection. Nosocomial infection may be defined as "clinical infection that develops after 48-72 hrs of admission to a hospital for treatment of a different initial illness, these infections are neither overtly present nor within the incubation period at the time of admission, and are often due to organisms endemic to the institution". ${ }^{7}$ As the characteristic red pigment production and the typical drug resistance pattern of S. marcescens speaks for itself, we did not opt for any molecular diagnosis.

\section{REFERENCES:}

1. Gaughran E.R., 1969. From superstition to science: the history of a bacterium. Trans NY Acad Sci, 31, 3-24.

2. Mackie \& McCartney, Practical Medical Microbiology, $14^{\text {th }}$ ed, 2012, p. 371.

3. Davis B.R., Ewing W.H. and Reavis R. W. 1957. The biochemical reaction given by members of the Serratia group. Int Bull Bacteriol Nomenclature Taxon, 7, 151-60.

4. Willium R.P., Green J. A. and Rappoport D.A. 1956. A study on pigmentation of Serratia marcescens, I. spectral and paper chromatographic properties of prodigiosin. J. Bacteriol, 71, 115-20.

5. Mandell GL, Bennett JE, Dolin R: Principles and Practices of Infectious Diseases, 7th edition, Philadelphia, 2010.

6. Difco Laboratories: Differentiation of Enterobacteriaceae by biochemical tests, Detroit, 1980.

7. Ananthanarayan \& Paniker's Textbook of Microbiology, 9th ed, 2013, P.644. 


\section{CASE REPORT}

\section{AUTHORS:}

1. Dipankar Paul

2. Indrani Bhattacharyya

3. Mandira Chakraborty

4. Palash Das

5. Sangita Das Ghosh

\section{PARTICULARS OF CONTRIBUTORS:}

1. Post Graduate Trainee, Department of Microbiology, CSTM, Kolkata.

2. Assistant Professor, Department of Microbiology, CSTM, Kolkata.

3. Post Graduate Trainee, Department of Microbiology, CSTM, Kolkata.

4. Post Graduate Trainee, Department of Microbiology, CSTM, Kolkata.
5. Post Graduate Trainee, Department of Microbiology, CSTM, Kolkata.

\section{NAME ADDRESS EMAIL ID OF THE CORRESPONDING AUTHOR:}

Dr. Indrani Bhattacharyya,

Assistant Professor,

Department of Microbiology,

Calcutta School of Tropical Medicine,

108, C. R. Avenue,

Kolkata - 700073.

Email: indranichaudhuri@yahoo.com

Date of Submission: 09/09/2014.

Date of Peer Review: 10/09/2014.

Date of Acceptance: 20/09/2014.

Date of Publishing: 01/10/2014. 